UDC: $530.145 .023: 531-4$

\title{
A CONCEPT OF UNIVERSAL QUANTUM JUMP
}

\author{
MAZEN KHODER
}

\begin{abstract}
It is known that quantum mechanics is one of the most successful theories in physics across the entire history of physics. Nevertheless, many believe that its foundations are still not really understood like: wave-particle duality, interference, entanglement, quantum tunneling, uncertainty principle, wave collapse, vacuum energy, relation between classical mechanics and quantum mechanics, classical limit, quantum chaos, etc., and the continuous failures in the unifying between relativity theory and quantum theory may be an indication about a problem in the foundations. This paper aims at discovering the first small step in the path of solving and understanding these quantum puzzles, in fact, the key to solving quantum puzzles is by understanding the reality of the motion and how it occurs. This paper proposes a model of motion that generalizes the concept of "quantum jump". Actually, we have been able to deduce the principles of quantum mechanics so that the oddity of the quantum puzzles becomes easier to understand and interpret. And as a direct verification of this model of motion this paper deduces the Lamb shift effect without the need of introducing of the concept of vacuum energy fluctuations, and it also discusses that the universal basic law of motion must be broader than both quantum mechanics and classical mechanics.
\end{abstract}

\section{INTRODUCTION}

It is known that the foundations of quantum mechanics are still not really understood. In the fifties of the last century began serious attempts to find an alternative theory of quantum mechanics or at least to understand its obsolescence and still this attempts continue until today, for example: David Bohm "Bohmian mechanics" [2, Hugh Everett "The Many-Worlds Interpretation of Quantum Mechanics" [7, Nelson "Stochastic Theory" [14], Gerhard Grossing "Nonequilibrium Thermodynamics" [9], Laurent Nottale "principles of scale relativity" [15], A. Bouda and Toufik Djama [4, 5, Faraggi and Matone [8, Antony Valentini "Dynamical origin of quantum probabilities" [18] and many others.

2010 Mathematics Subject Classification. Primary: 70A05; Secondary: 81P13, 81P05.

Key words and phrases. Mechanics of particles, quantum foundations, wave-particle duality, interference, entanglement, quantum tunneling, uncertainty principle, wave collapse, vacuum energy, relation between classical mechanics and quantum mechanics, classical limit, quantum chaos. 
It is known that the correspondence principle states that the behavior of systems described by quantum mechanics reproduces in a statistical way the classical mechanics in the limit of large quantum numbers, so because we have only a statistical matching in the classical limit between quantum mechanics and classical mechanics, Bohr stated that quantum mechanics does not produce classical mechanics in a similar way as the classical mechanics arises as an approximation of special relativity at velocities very slow than light speed. He argued that classical mechanics exists independently of quantum mechanics and cannot be derived from it. Max Jammer has said: "Quantum mechanics and classical dynamics are built on fundamentally different foundations"! [11. Many modern research [12, 16. confirms that quantum mechanics can not reproduce classical mechanics.

Based on this fact it seems that the general law of movement must be broader than both quantum mechanics and classical mechanics!

One important example is the particle in a box model (the infinite potential well) if we have the potential $V$ given by :

$$
V= \begin{cases}0, & 0<x<a \\ \infty, & x \leq 0, x \geq a\end{cases}
$$

$a$ is the length of the box, and $x$ is the position of the particle within the box, then the wave function for the stationary state is :

$$
\psi_{n}(x, t)=\sqrt{\frac{2}{a}} \sin \left(\frac{n \pi x}{a}\right) \exp \left(\frac{-i E_{n} t}{\hbar}\right),
$$

so the probability density for finding the particle is :

$$
P_{n}(x)=\frac{2}{a}\left|\sin \left(\frac{n \pi x}{a}\right)\right|^{2}
$$

and if $k=\frac{p}{\hbar}$ the probability density of momentum $p$ of the particle is :

$$
P_{n}(p)=\frac{a}{\pi \hbar}\left(\frac{n \pi}{n \pi+k a}\right)^{2} \operatorname{sinc}^{2}\left(\frac{1}{2}(n \pi-k a)\right) .
$$

We know that for large number $n$ we have:

$$
\lim _{n \rightarrow \infty} P_{n}(p)=\frac{1}{2}\left(\delta\left(p+\frac{n \pi \hbar}{a}\right)+\delta\left(p-\frac{n \pi \hbar}{a}\right)\right)
$$

so we arrived at the classical limit when the velocity is $\frac{n \pi \hbar}{a}$ for the same energy level. In this case, Einstein states [6] that the quantum mechanics is satisfactory complete for the momentum but it is not for the position because (based on the probability density for finding the particle) we have always some points where the particle can never exist.

If we examine the probability density for finding the particle when $n \rightarrow \infty$, we find a sequence of peaks separated by a distance equal to:

$$
\frac{a}{n}=\frac{\lambda}{2}
$$


where $\lambda$ is de Broglie wavelength:

$$
\lambda=\frac{h}{m v}
$$

and $v$ is the classical velocity. So, if the correspondence principle describes exactly the reality we need to oppose the objection of Einstein and affirm that in fact, the motion does not continue.

So we need to start from the concept of the motion itself. The motion as we know is related to space and time, it is a continuous change in position of a particle over time, but the existence of the particle in our world during its movement causes a real logical problem. It is about the continuity thus the infinity of a particle's positions, it corresponds to Zeno's paradoxes which were issued by the philosopher Zeno of Elea (ca. 490-430 BC). He claimed that "The first asserts the non-existence of motion on the ground that which is in locomotion must arrive at the half-way stage before it arrives at the goal. (Aristotle Physics, 239b11)" and "The [second] argument was called "Achilles", accordingly, from the fact that Achilles was taken [as a character] in it and the argument says that it is impossible for him to overtake the tortoise when pursuing it. (Simplicius(b) On Aristotle's Physics, 1014.10)" [10].

This being said, we must resolve this problem by either assuming that space is not continuous or the movement itself is not continuous. In fact, both assumptions must be taken into consideration.

This paper presents a theory of discontinuous motion of particles in continuous space-time.

So we start from the concept of the motion itself and assume that the motion (in the quantum world and classical world as well) is a sequence of appearances and disappearances events in space and time.

This is not the first time to assume such idea, some other scientists take this idea seriously.

Gao Shan presents a theory of discontinuous motion of particles [17, Laurent Nottale, Scale relativity [15] which is a geometrical and fractal space-time theory, Boisvert, Wilfrid, who has self-published his first book "Theory of Instantaneous Motion" [3].

In general, the earlier suppositions are good attempts which take the idea of discontinuity of motion as a real fact.

But according with the presented concept, it appears that there are certain criticisms in their works which briefly come as follows:

- Shan and Boisvert assume that the motion is spontaneous and it is not clear in their theories what is the link between the classical mechanic and quantum mechanic.

- Laurent Nottale declares that the motion is non-differentiable but it is continuous, and he uses the concept of velocity as a complex number. 


\section{The Motion}

Let's assume (FIG. 1.) that $\varepsilon$ is the duration during which a moving particle exists before disappearing and that $\mu$ is the duration of the particle's disappearance from our world before it reappears later.

So what about the trajectory of particle?

Since the particle's motion is a sequence of appearances and disappearances events, the continuous trajectory of the particle cannot exist, but we can suppose that for each disappearance and appearance events we have an imaginary path (FIG 1. ) that only reflects the properties of space and time on the values of $\varepsilon$ and

$\mu$.

If the particle at time $t_{1}$ appears in location $p_{1}$ and at time $t_{2}$ appears in location $p_{2}$ affected by an imaginary path, with velocity $v$, we can suppose:

$$
L=\int_{t_{1}}^{t_{2}} v d t .
$$

$L$ is the length of the imaginary path, and we have:

$$
t_{2}-t_{1}=\varepsilon+\mu .
$$

We can suppose that the particle did not measure the duration of its disappearance $\mu$ from our world simply because it was not in our world during this phase.

Therefore, we can suppose based on special relativity that:

$$
\tau=\int_{t_{1}}^{t_{2}} \sqrt{1-\frac{v^{2}}{c^{2}}} d t
$$

That $\tau$ is the proper time, we mean the time which the particle measured in its related reference during its movement from $\left(p_{1}, t_{1}\right)$ to $\left(p_{2}, t_{2}\right)$ using (or affecting by) an imaginary path. Note that this integral is a line integral where the function to be integrated is evaluated along a curve. So, we suppose that $\varepsilon=\tau$, then:

$$
\varepsilon=\int_{t_{1}}^{t_{2}} \sqrt{1-\frac{v^{2}}{c^{2}}} d t .
$$

If $v=$ const. or by using the mean velocity on path, then

$$
\begin{aligned}
& \varepsilon=\sqrt{1-\frac{v^{2}}{c^{2}}}(\varepsilon+\mu) \\
& \mu=\varepsilon \frac{\left(1-\sqrt{1-\frac{v^{2}}{c^{2}}}\right)}{\sqrt{1-\frac{v^{2}}{c^{2}}}} \\
& L=v(\varepsilon+\mu)
\end{aligned}
$$




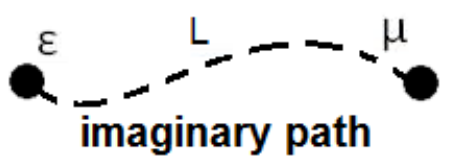

Figure 1. One quantum jump.

\section{Universal Quantum JumP}

First, we mean specifically by "universal quantum jump" (or we call it simply "quantum jump") one period of movement between two appearances of a particle. So the quantum jump is not related only when the particle moves from one energy level to another, but also the particle makes jump when it moves from one position to another.

As we know, based on Newton's First law of motion:

"In an inertial reference frame, an object either remains at rest or continues to move at a constant velocity, unless acted upon by a force".

But this law is not compatible with the disappearance and appearance idea, since the particle might easily appear in a forbidden (have a variation to a very large potential field like for example particle in a box) place after some quantum jumps in the direction of the movement of the particle (because in Newton mechanics, the particle don't take into consideration the potential energy that is far from its current location) so for a huge number of particles that jump in the subatomic level, the Newton law may put our universe in an unstable situation, and this might happen specifically when the length of the jump is close (or greater) to the length of the field's fluctuations.

But in the case where the length of the quantum jump is very small compared to the length of the field's fluctuations, then the first law of Newton will be applicable because in this case, we can be sure that the particle will feel the force before that the force gets altered so all initial velocities are acceptable.

In this case (classical world) if the initial velocity is $\vec{v}$ then this velocity must be constant during one quantum jump (because there is no significant change in the potential field), and we assume that the quantum jump $J$ should be (in non-relativistic case) the half of the de Broglie wavelength:

$$
J=\frac{\lambda}{2}=\frac{1}{2} \frac{h}{m v}
$$

Therefore, we need to add half of the Compton wavelength to take in consideration the relativistic effect, so finally, we suppose (in classical world) that:

$$
J=\frac{h}{2 m v}+\frac{h}{2 m_{0} c}
$$

$m_{0}$ is the rest mass or invariant mass $\Rightarrow$

$$
L=\frac{h}{2 m v}+\frac{h}{2 m_{0} c}
$$




$$
\begin{aligned}
& \varepsilon=\left(\frac{h}{2 m v^{2}}+\frac{h}{2 m_{0} c^{2}}\right) \sqrt{1-\frac{v^{2}}{c^{2}}} \\
& \mu=\left(\frac{h}{2 m v^{2}}+\frac{h}{2 m_{0} c^{2}}\right)\left(1-\sqrt{1-\frac{v^{2}}{c^{2}}}\right)
\end{aligned}
$$

when $v \ll c \Rightarrow$

when $v \rightarrow c$

$$
L=\frac{h}{2 m v}, \varepsilon=\frac{h}{2 m v^{2}}, \quad \mu=\frac{h}{4 m c^{2}}
$$

$$
L=\frac{h}{2 m_{0} c}, \varepsilon=0, \mu=\frac{h}{2 m_{0} c^{2}}
$$

We know that $\vec{F}=m \vec{\gamma}$ but in the point of view of universal quantum jump theory the real Newton equation must not contain the derivative of velocity (unless as an approximation) because the motion always must be a sequence of quantum jumps, so in the classical word the Newton equation become (for example when $v \ll c$ ):

$$
\begin{gathered}
\vec{F} \varepsilon_{2}=m \vec{v}_{2}-m \vec{v}_{1} \\
\varepsilon_{2}=\frac{h}{2 m v_{2}^{2}}
\end{gathered}
$$

So, in classical world when $\vec{v}_{1}$ is the initial velocity we can calculate $\vec{v}_{2}$ that is the new velocity and $\varepsilon_{2}$ that is the duration of existence of the particle in our world before disappearing.

But when we come out from classical phase and enter to the quantum phase we need to modify Newton's first law as follows: In any reference, an object either remains at rest or continues to move using a quantum jump based on a new action principle called "alike action principle" that takes in consideration all forces existent in the universe (not only the applicable forces on the particle itself).

\section{Alike action principle}

When the particle is in location $p_{1}$ at time $t_{1}$ and we are investigating where would it be in time $t_{2}$ ?

We use this equation to distinguish all space paths:

$$
L=\int_{t_{1}}^{t_{2}} v d t
$$

for each path, we can define the ordinary action $\mathrm{S}$ which is verified by:

$$
S=\int_{t_{1}}^{t_{2}}\left(\frac{1}{2} m v^{2}-U\right) d t
$$

$U$ is the potential energy, now since we have a lot of choices for the location in time $t_{2}$, we suppose that we are in quantum phase or in other words. We ignore the classical mechanic effect which permits us to ignore the initial velocity (for the imaginary path of a particle used to come to the initial position $p_{1}$ ).

In our case (quantum phase) the initial velocity didn't have a real significant effect on the movement of the particle as if the particle always forgets how it came 


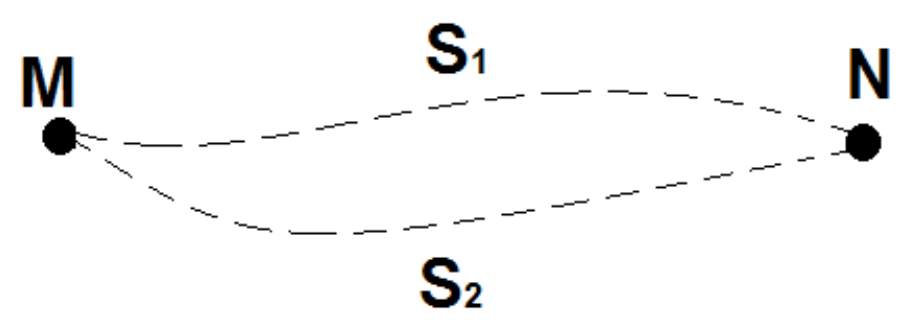

FiguRE 2. Two imaginary paths.

to its initial position and starts again without any initial velocity.

In the quantum phase the particle has some preferred destinations based on a new quantum action principle named "alike action principle" that ensures the existence of physical harmony within our universe, like for example preventing the particle from easily reaching to forbidden locations (guarded by fields of great forces).

Therefore, in general, this new constraint in movement could be valid at multiple positions at the same time, so in general, we have multiple acceptable positions in time $t_{2}$. Thus the probability of existence came up in our description of the movement in quantum world.

We suppose that we have a preferred value of action that we call $\mathrm{h}$ (Plank constant), the new action principle called "alike action principle" states:

"The preferred appearance destination took by the particle at time $t$ is the one for which all the remainders due to $\frac{S}{h}$ (for all paths which lead to this destination) are stationary".

In other words, it is as having the same (or close to each other) remainder after dividing them by $\mathrm{h}$.

For example, if we have two actions (for two paths) to one destination location (FIG. 2.):

$$
\begin{aligned}
& S_{1} \text { and } S_{2} \Rightarrow S_{1}=n_{1} h+r_{1} h, 0<r_{1}<1 \\
& \text { and } S_{2}=n_{2} h+r_{2} h, 0<r_{2}<1 \Rightarrow \\
& S_{2}-S_{1}=\left(n_{2}-n_{1}\right) h+\left(r_{2}-r_{1}\right) h
\end{aligned}
$$

and if we have $r_{2}-r_{1}=0$ then $S_{2}$ and $S_{1}$ have the same remainder after dividing by $h$ and then we have a preferred location (point $\mathrm{N}$ ) at time $t_{2}$, but if we have $\left|r_{2}-r_{1}\right|=\frac{1}{2}$ then the difference between the remainders reach its maximum so we have a forbidden location (point $\mathrm{N}$ ) at time $t_{2}$. So, we can say that the action value $h$ is the action preferred in nature and it is the preferred unit of quantum jump and in reverse, it becomes not preferable as it goes far from $h$.

We need to find a function which verifies the following requirements: $f(n h)=0$ and $f\left(n h+\frac{h}{2}\right)=1$ (using as maximum).

It obvious that this function is a periodic function and the most simple one that verifies these requirements is:

$$
\sin ^{2}(x)
$$




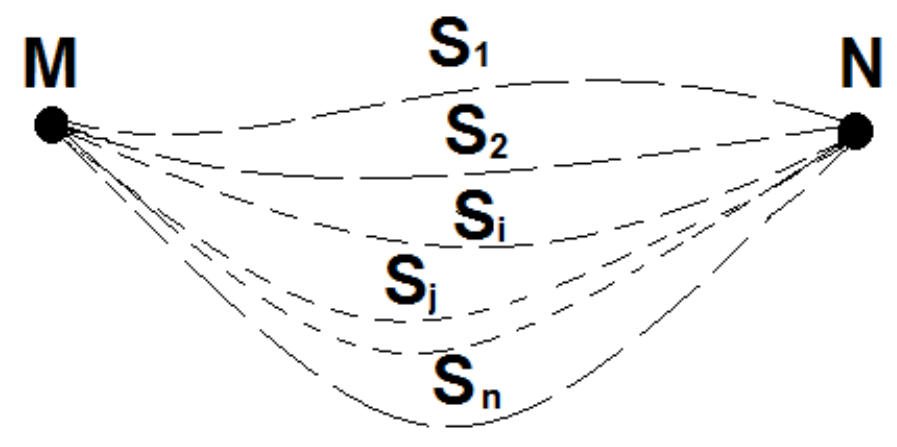

FiguRE 3. Many imaginary paths.

Thus by having two actions $S_{1}$ and $S_{2}$ we can define the "difference quantum actions" as:

$$
\delta_{S_{1}, S_{2}}=\sin ^{2}\left(\frac{\pi}{h}\left(S_{1}-S_{2}\right)\right)
$$

So when the difference of quantum actions is at minimum it yields a maximum preferred destination of particle and when we have a maximum difference of quantum actions it yields to a minimum preferred destination of particle and down to forbidden destination.

If we have $n$ paths (In real case we have infinities of paths) to the potential destination of the particle (FIG. 3.) then we can simply suppose that the difference quantum actions for all $n$ paths are the sum of all the differences between each pair of paths:

$$
\delta_{S_{1}, S_{2}, \ldots S_{n}}=\sum_{(i, j)} \sin ^{2}\left(\frac{\pi}{h}\left(S_{i}-S_{j}\right)\right)
$$

We have some important mathematical properties for the equation (4.3) which we introduce here without demonstrations:

$$
\begin{gathered}
\max \text { of }\left\{\delta_{S_{1}, S_{2}, \ldots S_{n}}\right\}=\frac{n^{2}}{4} \\
\lim _{\left(S_{i}-S_{j} \bmod h\right) \text { is equiprobable in }[0, \mathrm{~h}]} \delta_{S_{1}, S_{2}, \ldots S_{n}}=\frac{n(n-1)}{4}
\end{gathered}
$$

\section{Derivation of the Path integral formulation}

We now want to specify the equation which can be used to calculate the quantity $Q$ that is proportional to the probability of existence of any potential destinations at time $t_{2}$, so for each potential destination the input of this function is $\delta_{S_{1}, S_{2}, \ldots S_{n}}$ and the output is proportional to the probability of appearance of the particle in this destination. Accordingly, this probability is maximum when $\delta_{S_{1}, S_{2}, \ldots S_{n}}=0$ and it is minimum (zero) when $\delta_{S_{1}, S_{2}, \ldots S_{n}}$ is maximum, i.e. when $\delta_{S_{1}, S_{2}, \ldots S_{n}}=\frac{n^{2}}{4}$ and we can also assume in the case of equation 4.5 that the distribution of the quantum difference of actions is allocated evenly across the range between 0 and 
$h$.

There isn't any tendency to forbid reaching to the destination nor to maximize the probability of appearance of the particle at this destination, therefore, in this case, we can suppose that the probability of appearance is proportional to the number of all paths (like classical case).

We can simply suppose that the function is linear to the $\delta_{S_{1}, S_{2}, \ldots S_{n}}$ so:

$$
\begin{aligned}
Q & =a \delta_{S_{1}, S_{2}, \ldots S_{n}}+b, \mathrm{a} \text { and } \mathrm{b} \text { are constants } \Rightarrow \text { we have: } \\
0 & =a \frac{n^{2}}{4}+b, \text { and } n=a \frac{n(n-1)}{4}+b \Rightarrow \\
Q & =n^{2}-4 \delta_{S_{1}, S_{2}, \ldots S_{n}}
\end{aligned}
$$

Ultimately we have three main results:

One which could be reduced to the classical mechanic (in this case all $\mathrm{n}$ paths are acceptable), and the two others are pure quantum results in which one of them increases the probability of appearance to become proportional to $n^{2}$ and the other prevents any appearance in the selected destination.

It becomes clear that the hidden variable in quantum phase is the quantum jump itself which will be taken by the particle during the next jump based on a new action principle called "alike action principle", so we understand the origin of the nonlocality of the quantum hidden variable as we know it for example in the Kochen-Specker theorem [13].

Now we want to verify that this quantum "alike action principle" yields to the path integral formulation of quantum mechanics which is equivalent to Schrodinger equation, so from equation (5.1) we have that:

$$
\begin{aligned}
Q & =n^{2}-4 \delta_{S_{1}, S_{2}, \ldots S_{n}} \Rightarrow \text { if we take } n=2 \Rightarrow \\
Q & =4-4 \delta_{S_{1}, S_{2}} \\
& =4-4 \sin ^{2}\left(\frac{\pi}{h}\left(S_{1}-S_{2}\right)\right) \\
& =2\left(1+\cos \left(\frac{2 \pi}{h}\left(S_{1}-S_{2}\right)\right)\right) \\
& =\cos ^{2}\left(\frac{2 \pi}{h} S_{1}\right)+\sin ^{2}\left(\frac{2 \pi}{h} S_{1}\right)+\cos ^{2}\left(\frac{2 \pi}{h} S_{2}\right)+\sin ^{2}\left(\frac{2 \pi}{h} S_{2}\right) \\
& +2 \cos \left(\frac{2 \pi}{h} S_{1}\right) \cos \left(\frac{2 \pi}{h} S_{2}\right)+2 \sin \left(\frac{2 \pi}{h} S_{1}\right) \sin \left(\frac{2 \pi}{h} S_{2}\right) \\
& =\left(\cos \left(\frac{2 \pi}{h} S_{1}\right)+\cos \left(\frac{2 \pi}{h} S_{2}\right)\right)^{2}+ \\
& \left(\sin \left(\frac{2 \pi}{h} S_{1}\right)+\sin \left(\frac{2 \pi}{h} S_{2}\right)\right)^{2} \\
& =\left|\exp \left(i \frac{2 \pi}{h} S_{1}\right)+\exp \left(i \frac{2 \pi}{h} S_{2}\right)\right|^{2} .
\end{aligned}
$$

So, in general we can prove:

$$
Q_{S_{1}, S_{2}, \ldots S_{n}}=\left|\sum_{i=1}^{n} \exp \left(i \frac{2 \pi}{h} S_{i}\right)\right|^{2}
$$


so, we derive the "path integral formulation" of quantum mechanics discovered by Feynman (for infinity paths the sum become an integral).

Thus, when the particle exists in a location $M$ in time $t_{1}$ we need to apply the quantum "alike action principle" in all locations to find the probability of appearance at time $t_{2}$ which is proportional to the quantity $Q_{S_{1}, S_{2}, \ldots S_{n}}$ in each location. So, any modification in these locations, for example by modifying the fields through which the imaginary paths go through will affect the calculation of $Q_{S_{1}, S_{2}, \ldots S_{n}}$.

5.1. Role of space-time in motion. In summary we usually deal with the motion like it was related only with the particle itself, but based on the universal quantum jump, this is not true, so we have two players in the motion:

1- The particle itself

2- The space-time itself

At each time, space itself allows the particle to appear in some multiple positions with certain preferences based on the new quantum action principle "alike action principle" that ensures the existence of physical harmony within our universe, and the particle chooses randomly between these preferences.

So, this is the role of space-time in the motion process, like for example preventing the particle from easily reaching to forbidden locations (guarded by fields of great forces).

Therefore, in general, this new constraint in movement could be valid at multiple positions at the same time, so in general, we have multiple acceptable positions to appear at it, thus the probability of existence came up in our descriptions of the movement in the quantum world.

5.2. Case of photon. For photon we can do similar to the particle, so when $\lambda$ much smaller than the scale of slits etc., then the photon follows a direct line with the quantum jump in equation (3.1) for velocity equals $c$, but when $\lambda$ is comparable or greater then the scale of slits for example, then the photon follow its "alike action principle".

So, for $S=\int_{t_{1}}^{t_{2}}\left(\frac{1}{2} m v^{2}-U\right) d t$, the similar quantity of $\frac{1}{2} m v^{2}$ is the kinetic energy $E_{k}=m c^{2}-m_{0} c^{2}$ so for photon we can say

$$
\begin{aligned}
& m_{0} c^{2}=0 \Rightarrow E_{k}=m c^{2} \Rightarrow \\
& \frac{S}{h}=\frac{m c^{2}\left(t_{2}-t_{1}\right)}{h} \Rightarrow \frac{S}{h}=\frac{L}{\lambda}, \mathrm{L} \text { is the path length } \Rightarrow \\
& Q_{L_{1}, L_{2}, \ldots L_{n}}=\left|\sum_{i=1}^{n} \exp \left(i \frac{2 \pi}{\lambda} L_{i}\right)\right|^{2}
\end{aligned}
$$

\section{Heuristic DERIVATION OF LAMB SHifT}

Currently, we know that the Lamb shift happened by the interaction between vacuum energy fluctuations and the hydrogen electron, to derive it we need the technique of renormalization that we use in quantum electrodynamics theory.

But we can do that without referring to vacuum energy at all, or more exactly 


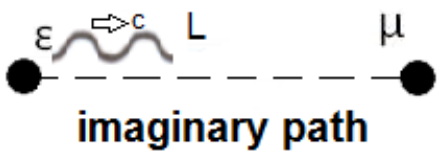

Figure 4. The electron quantum jump

we can understand from where come the vacuum energy fluctuations and what it is in fact.

We know before from equations (2.4) and 2.5) that if $v \simeq$ constant or if we use the mean of velocity, then

$$
\begin{aligned}
& \varepsilon=\sqrt{1-\frac{v^{2}}{c^{2}}}(\varepsilon+\mu) \\
& \mu=\frac{\varepsilon\left(1-\sqrt{1-\frac{v^{2}}{c^{2}}}\right)}{\sqrt{1-\frac{v^{2}}{c^{2}}}} \\
& L=v(\varepsilon+\mu)
\end{aligned}
$$

So, if $v \ll c$ then:

$$
\frac{L}{\mu}=\frac{2 c^{2}}{v}
$$

Then the real velocity of quantum jump exceeds the speed of light (but always the apparent velocity or the mean velocity is less than the speed of light), meaning that it exceeds the speed of the electromagnetic wave.

So, after the electron jumps it feels some electromagnetic waves (virtual photons) that were sent by him before it disappears, in other words, it sees itself like another electron that reacts with it.

If $d$ is the duration while some part of emitted electromagnetic waves from the electron arrive on the electron itself after it jumps, and if we work on the direct line $L$ between the old position of the electron and the new position, as it is the imaginary path of it then: (please see FIG. 4):

$$
\begin{aligned}
& L=c d+c \mu \\
& d=\frac{L-c \mu}{c} \\
& d=\varepsilon \frac{\sqrt{1-\frac{v^{2}}{c^{2}}}+\frac{v}{c}-1}{\sqrt{1-\frac{v^{2}}{c^{2}}}}
\end{aligned}
$$

So if we ignore the relativistic correction then for $v \ll c$ : 


$$
d=\frac{\varepsilon v}{c}
$$

So, the real duration of interaction is equal the ordinary duration $\varepsilon$ multiply by new factor $\frac{v}{c}$ then we need to multiply the ordinary coulomb force with the same factor, therefore the electron push itself and move it slightly away from the proton, then the electrostatic force that feels the electron from itself is:

$$
\frac{1}{4 \pi \varepsilon_{0}} \frac{e^{2}}{r^{2}} \frac{v}{c}=m \frac{d v}{d t}
$$

And $r=L$ is the direct distance between the old position of the electron and the new one just after the quantum jump, then the $d t$ is the real duration of interaction $d$ then:

$$
\begin{aligned}
& d t=d=\frac{\varepsilon v}{c} \text { and: } \\
& L=\varepsilon v=r \Rightarrow \\
& d t=\frac{r}{c} \Rightarrow \\
& \frac{1}{4 \pi \varepsilon_{0}} \frac{e^{2}}{r} \frac{1}{m c} \frac{v}{c}=d v
\end{aligned}
$$

For the ground state, we can (as an approximation) replace the velocity $v$ by the value of the Bohr velocity of the electron in its first orbit (the ground state) so we can suppose that:

$$
v=\frac{\hbar}{m r}
$$

Finally to calculate the tiny displacement caused by this force we need to multiply $d v$ by $d t$ therefore we have:

$$
\begin{aligned}
& d r_{r}=\frac{1}{4 \pi \varepsilon_{0}} \frac{e^{2}}{r m c} \frac{v}{c} \frac{r}{c} \Rightarrow \\
& d r_{r}=\frac{1}{4 \pi \varepsilon_{0}} \frac{e^{2}}{r m c} \frac{\hbar}{m r c} \frac{r}{c} \Rightarrow \\
& d r_{r}=\frac{1}{4 \pi \varepsilon_{0}} \frac{e^{2} \hbar}{m^{2} c^{3}} \frac{1}{r} \Rightarrow \\
& |d r|_{r}^{2}=\frac{1}{4 \pi \varepsilon_{0}} \frac{e^{2} \hbar}{m^{2} c^{3}} \frac{d r}{r} \Rightarrow \\
& (d r)^{2}=\sum|d r|_{r}^{2}=\frac{1}{4 \pi \varepsilon_{0}} \frac{e^{2} \hbar}{m^{2} c^{3}} \int \frac{d r}{r}
\end{aligned}
$$

For the ground state, the electron probability density has a peak at the middle of the atom and go around it with a distance equal to Bohr radius:

$$
a_{0}=\frac{4 \pi \varepsilon_{0} \hbar^{2}}{m e^{2}}
$$


so, $r$ must be less than $a_{0}$, and when $r$ become very small compared with $a_{0}$ we can use the classical case and suppose that the quantum jump follows the equation (3.1.

$$
L=r=\frac{h}{2 m v}+\frac{h}{2 m_{0} c} \Rightarrow r>\frac{h}{2 m_{0} c} .
$$

Finally, we can derive the perturbation in the position of the electron that explains the energy shift:

$$
\begin{aligned}
& (d r)^{2}=\sum|d r|_{r}^{2}=\frac{1}{4 \pi \varepsilon_{0}} \frac{e^{2} \hbar}{m^{2} c^{3}} \int_{\frac{h}{2 m_{0} c}}^{a_{0}} \frac{d r}{r} \Rightarrow \\
& (d r)^{2} \sim \frac{e^{2} \hbar}{m^{2} c^{3}} \ln \frac{4 \varepsilon_{0} \hbar c}{e^{2}}
\end{aligned}
$$

We can use another approach by simply follow the steps in textbook with identifying that the origin of virtual photons is the electron itself before it did its jump. As we see, this paper can give us the Lamb shift without need the concept of vacuum fluctuation, so based on this idea the vacuum fluctuations are just an illusion, in fact, this is simply the action of the particle on itself right after the quantum jump happened.

\section{DISCUSSIONS AND CONCLUSIONS}

So, the observer didn't decide where the particle will appear but the particle itself has decided its next location among all possible destinations based on a new action principle called "alike action principle".

This clarifies to us and makes more understandable all strange behaviors of matter in quantum mechanics such as interference, wave collapse, entanglement, quantum tunneling, uncertainty principle etc.

7.1. Wave collapse. The appearance of a particle in any new position will lead once again to the calculation of quantum alike action which clarifies what is called wave collapse. So, we can conclude that the wave collapse it is simply the appearance event of the particle in space. Furthermore, it also clarifies the "decoherence" concept which illustrates the effect of the environment on the wave function when the particle chooses one location (when it interacts with the environment) to appear.

So, the Einstein's supposition that the observation is not related to the observer, as well as Bohr's supposition that the observer causes the observation do not hold in this framework, namely, here, the observed particle itself causes the observation by choosing one location to appear, based on the quantum "alike action principle".

7.2. Entanglement. For instance, regarding the entanglement, in fact, two entangled particles took those related physical values (as the conservation law states) in coordination while being adjacent and we observed this physical values right 
after their quantum jump which has occurred at a jump velocity:

$$
v_{J}=\frac{L}{\mu}
$$

In case when $v \ll c$ then $v_{J}$ should exceed the speed of light so we do not have any "spooky action at a distance", for example of equations (2.5) if $v \simeq$ constant or if we use the mean velocity $\Rightarrow$

$$
L=v\left(t_{2}-t_{1}\right), \mu=\frac{v^{2}\left(t_{2}-t_{1}\right)}{2 c^{2}} \Rightarrow v_{J}=\frac{L}{\mu}=\frac{2 c^{2}}{v}
$$

7.3. Compatibility with special relativity. As we see in the previous subsection the particle can do a jump with $v_{J}$ exceeding the speed of light, so is this causes an inconsistency between frames of reference, that leads to violation of the law of energy conservation.

For example, if we have one particle, according to one frame of reference the particle did one jump but for another frame of reference observe the two particles at the same time instead of seeing also the jump of the particle. First, we need to define exactly what we mean by "according to one frame of reference ....we observe...", one frame observes something, for example by sending photon to it, so we need some interaction between the frame and the system.

Now, the important thing is what happens when we observe the particle?

When we observe the particle the duration $\varepsilon$ simply will start, that means if we observe the particle again (before it disappears) the duration $\varepsilon$ starts again and this idea is compatible with "Quantum Zeno effect", this effect is interpreted as "a system can't change while you are watching it".

So now, the jump is always compatible with special relativity because always we see $v<c$, we can't see $v_{J}$, in other words, we can't see the exact moment of the jump, we always see the start moment of the duration of $\varepsilon$.

7.4. Basic law of motion. Based on the idea of disappearance and appearance it seems that the general equation of motion is a combination of the quantum "alike action principle" (equation (5.2) ) and the quantified Newton law of motion (equations (3.4 3.5) so when the quantum jump is very small compared to the length of the potential field's fluctuations then the particle takes into consideration the initial velocity $v$ and follows the quantified Newton law of motion (equations (3.4 3.5).

On the contrary, when the length of the jump is close (or greater) to the length of the potential field's fluctuations then the particle ignores the initial velocity $v$ or its effect becomes minimal effect and only uses the quantum "alike action principle" to know where it will go.

So, in classical limit, i.e. when the particle enters in the classical regime, the initial velocity takes its role to specify the future movement, in this case we may witness a chaotic behavior (big sensitivity to initial values) when we put for example the particle in a special shape such as a stadium billiard. 
In parallel, when we refer back to the quantum regime the particle always ignores the initial velocities. Thus, the chaos will disappear from the quantum world (we verified Berry [1]) as we know in quantum chaos because the Schrodinger equation is a linear equation which doesn't consider a chaotic behavior as Newton law does.

\section{REFERENCES}

[1] M. Berry, Quantum physics on the edge of chaos, New Scientist 19, November 1987, 44-47.

[2] D. Bohm, A Suggested Interpretation of the Quantum Theory in Terms of "Hidden" Variables, Physical Review, 85 (2), (1952), 166-179.

[3] W. Boisvert, Theory of Instantaneous Motion, self-published, 1953.

[4] A. Bouda, T. Djama, Quantum Newton's Law, Physics Letters A, 285(1-2), (2001), 27-33.

[5] A. Bouda, T. Djama, Trajectories in the context of the quantum Newton's law, Phys. Scr. 66 (2), (2002), 97-104.

[6] A. Einstein, Scientific Papers Presented to Max Born on his retirement from the Tait Chair of Natural Philosophy in the University of Edinburgh, Oliver \& Boyd, 1953.

[7] H. Everett, On the Foundations of Quantum Mechanics, Ph.D. Thesis, Princeton University, Department of Physics, 1957.

[8] A. E. Faraggi, M. Matone, The Equivalence Postulate of Quantum Mechanics, International Journal of Modern Physics A, 15 (13), (2000), 1869-2017.

[9] G. Grossing, On the Thermodynamic Origin of the Quantum Potential, Phys.A, 388(6), (2009), 811-823.

[10] N. Huggett, Zeno's paradoxes, The Stanford Encyclopedia of Philosophy (Winter 2019 Edition), Edward N. Zalta (ed.),

https://plato.stanford .edu/archives/win2019/entries/paradox-zeno/

[11] M. Jammer, The Conceptual Development of Quantum Mechanics, McGraw-Hill, New York, 1966.

[12] U. Klein, What is the limit $\hbar \rightarrow 0$ of quantum theory?, American Journal of Physics 80 (11), (2012), 1009-1016.

[13] S. Kochen, E. P. Specker, The problem of hidden variables in quantum mechanics, Journal of Mathematics and Mechanics, 17 (1), (1967) 59-87.

[14] E. Nelson, Derivation of the Schrodinger Equation from Newtonian Mechanics, Phys. Rev. 150 (1966), 1079-1085.

[15] L. Nottale, Scale Relativity, Fractal Space-Time, and Quantum Mechanics, Chaos, Solitons \& Fractals, 4 (3), (1994), 361-388.

[16] M. Rabinowitz, Is Quantum Mechanics Incompatible with Newton's First Law?, Int. J. Theor. Phys. 47, (2008), 936-948.

[17] G. Shan, Interpreting Quantum Mechanics in Terms of Random Discontinuous Motion of Particles, Ph.D. Thesis, University of Sydney, Australia, Department of Philosophy, 2012.

[18] A. Valentini, H. Westman Dynamical origin of quantum probabilities, Proc. R. Soc. A (2005) $461,253-272$.

Lebanese University,

Faculty of Engineering, Lebanon

Email address: mazen.khoder@gmail.com

Received: 24.1 .2020

Revised: 15.5 .2020

Accepted: 25.5 .2020 